\title{
Relacionamentos amorosos e seus constituintes para a felicidade
}

\section{Loving relationships and their constituents for happiness}

\section{Las relaciones amorosas y sus componentes para la felicidad}

Cristina Soares Oliveira

ORCID: https://orcid.org/0000-0002-4917-6811 Centro Universitário de Ciências e Tecnologia do Maranhão, Brasil E-mail: csoaresoliveira27@gmail.com Kaio Germano Sousa da Silva ORCID: https://orcid.org/0000-0003-4236-6230 Faculdade do Vale Elvira Dayrell, Brasil E-mail: kaiogsds@hotmail.com Janaína Oliveira Morais

ORCID: https://orcid.org/0000-0002-4315-2691 Centro Universitário de Ciências e Tecnologia do Maranhão, Brasil E-mail: oliveira.janana@ hotmail.com

Edilene Ferreira da Costa Santos ORCID: https://orcid.org/0000-0001-7304-9401 Centro Universitário de Ciências e Tecnologia do Maranhão, Brasil E-mail: dilla.soares@hotmail.com

Francilene Regina Brito da Silva

ORCID: https://orcid.org/0000-0002-4323-1402 Centro Universitário de Ciências e Tecnologia do Maranhão, Brasil E-mail: francilenebrito@outlook.com

Ana Cristina Jácome Castelo Gomes

ORCID: https://orcid.org/0000-0002-3098-2872 Centro Universitário de Ciências e Tecnologia do Maranhão, Brasil E-mail: anajacomebb4@gmail.com

Alessandra Ferreira das Chagas ORCID: https://orcid.org/0000-0002-2407-0666 Centro Universitário de Ciências e Tecnologia do Maranhão, Brasil

E-mail: 18alenzo@gmail.com

Raquel Ferreira Coelho

ORCID: https://orcid.org/0000-0003-2988-544X Universidade Federal do Piauí, Brasil E-mail: raquelxcoelho@hotmail.com

Érika Castelo Branco Said

ORCID: https://orcid.org/0000-0003-1152-3424 Faculdade Inspirar, Brasil

E-mail: erikacbsaid@hotmail.com

Anastácia Tavares da Silva

ORCID: https://orcid.org/0000-0001-5106-4926

Centro Universitário de Ciências e Tecnologia do Maranhão, Brasil E-mail: anastaciapedrolulu@gmail.com

José Alberto Araújo dos Santos

ORCID: https://orcid.org/0000-0003-4434-5900

Centro Universitário de Ciências e Tecnologia do Maranhão, Brasil E-mail: jose-alberto980@ hotmail.com

Mariana Melo Machado

ORCID: https://orcid.org/0000-0002-3535-1375

Centro Universitário de Ciências e Tecnologia do Maranhão, Brasil E-mail: Marymmachadoo@gmail.com

Conceição Maria Melo Miranda

ORCID: https://orcid.org/0000-0002-9026-0222

Centro Universitário de Ciências e Tecnologia do Maranhão, Brasil

E-mail: Conceicaomirandda@gmail.com

Kayle Rodrigues dos Santos

ORCID: https://orcid.org/0000-0001-8343-5621

Centro Universitário de Ciências e Tecnologia do Maranhão, Brasil E-mail: kaylepsicologo18@gmail.com

Andreza da Conceição Araújo

ORCID: https://orcid.org/0000-0002-4668-8133

Centro Universitário de Ciências e Tecnologia do Maranhão, Brasil E-mail: andrezaaraujo88@gmail.com 


\title{
Valdileno Silva Mendes \\ ORCID: https://orcid.org/0000-0001-6295-0122 Centro Universitário de Ciências e Tecnologia do Maranhão, Brasil E-mail: valdilenomendes2@gmail.com \\ Francisca Tatiana Dourado Gonçalves ORCID: https://orcid.org/0000-0001-5414-0381 Universidade Luterana do Brasil, Brasil E-mail: tatyanadourado@yahoo.com.br
}

\begin{abstract}
Resumo
A Psicologia Positiva lida com a felicidade, funcionamento humano ótimo, focando nas características da personalidade positiva, no bem-estar e satisfação com a vida. Caracteriza a personalidade do indivíduo através das virtudes e forças de caráter que por sua vez, podem contribuir de forma considerável para a felicidade nos relacionamentos amorosos. O presente estudo teve como principal objetivo compreender a construção da personalidade na perspectiva da Psicologia Positiva e de que maneira os atributos de personalidade são favoráveis para a construção de bem-estar e satisfação nos relacionamentos amorosos. Trata-se de uma pesquisa bibliográfica do tipo revisão narrativa, descritiva e exploratória com abordagem qualitativa. Os fenômenos do otimismo, espiritualidade e criatividade, contribuem para uma compreensão dinâmica da personalidade e articulam-se como fatores de proteção e promoção para um relacionamento mais equilibrado e satisfatório. De acordo com os achados da pesquisa, é possível afirmar que os constructos da personalidade apresentados pela Psicologia Positiva denominados virtudes e forças de caráter são capazes de contribuir de forma considerável para o estabelecimento de bem-estar e qualidade na relação amorosa.
\end{abstract}

Palavras-chave: Psicologia positiva; Personalidade; Relacionamento conjugal; Felicidade.

\begin{abstract}
Positive psychology deals with happiness, excellence and optimal human functioning, focusing on positive personality characteristics, well-being and life satisfaction. It characterizes the personality of the individual through the virtues and forces of character that in turn can contribute considerably to happiness in loving relationships. The main objective of this study was to understand the construction of personality from the perspective of positive psychology and how personality attributes are favorable for the construction of well-being and satisfaction in loving relationships. This is a bibliographical research of the narrative, descriptive and exploratory review type with a qualitative approach. The phenomena of optimism, spirituality and creativity contribute to a dynamic understanding of personality and are articulated as protective and promotion factors for a more balanced and satisfactory relationship. According to the research findings, it is possible to affirm that the personality constructs presented by positive psychology called virtues and forces of character are able to contribute considerably to the establishment of well-being and quality in the love relationship.
\end{abstract}

Keywords: Positive psychology; Personality; Marital relationship; Happiness.

\section{Resumen}

La Psicología Positiva se ocupa de la felicidad, el funcionamiento humano óptimo, centrándose en los rasgos positivos de la personalidad, el bienestar y la satisfacción con la vida. Caracteriza la personalidad del individuo a través de las virtudes y fortalezas de carácter que, a su vez, pueden contribuir considerablemente a la felicidad en las relaciones amorosas. El objetivo principal de este estudio fue comprender la construcción de la personalidad desde la perspectiva de la Psicología Positiva y cómo los atributos de la personalidad son favorables para la construcción del bienestar y la satisfacción en las relaciones amorosas. Se trata de una revisión de la literatura del tipo de revisión narrativa, descriptiva y exploratoria con enfoque cualitativo. Los fenómenos de optimismo, espiritualidad y creatividad contribuyen a una comprensión dinámica de la personalidad y se articulan como factores de protección y promoción para una relación más equilibrada y satisfactoria. Según los hallazgos de la investigación, es posible afirmar que los constructos de personalidad presentados por la Psicología Positiva denominados virtudes y fortalezas de carácter son capaces de contribuir considerablemente al establecimiento de bienestar y calidad en la relación amorosa.

Palabras clave: Psicología positiva; Personalidad; Relación conyugal; Felicidad.

\section{Introdução}

A Psicologia Positiva é o estudo científico dos fatores e processos que levam à otimização da função humana, focando na força em vez da fraqueza. Além disso, constrói e consolida as melhores coisas da vida, em vez de tentar consertar o pior, e se preocupa em promover o estilo de vida mais benéfico entre as pessoas comuns, em vez de se concentrar no processo de cura das pessoas doentes (Nunes, 2007).

O bem-estar pode ser considerado um dos fatores mais estudados e abordados na Psicologia Positiva. Segundo 
Seligman (2012), o tema da Psicologia Positiva é o bem-estar, cujo principal critério de mensuração é o florescimento, por isso o foco da Psicologia Positiva é aumentar esse florescimento. Ainda de acordo com esse autor, é inegável que relacionamentos positivos ou a falta deles têm um efeito profundo na felicidade. Assim, pode-se tratar da importância da promoção do bem estar quando o assunto é relacionamento.

Há muitas definições para o termo felicidade, Figueiras (2017) afirma que as condições de felicidade ou bem-estar psicológico estão diretamente relacionadas à subjetividade do indivíduo ou à relação com os outros e com o meio ambiente. Pode haver outros fatores de felicidade que vão além, como a saúde e a ausência de doenças ou condições ambientais adversas. No entanto, há pessoas que podem ser mais resilientes diante de situações desafiadoras, assim como existem pessoas que, mesmo em boas condições, estão sempre insatisfeitas consigo mesmas e com os demais.

A Psicologia Positiva sugere medidas para compreender a felicidade por meio da compreensão das emoções positivas, engajamento, sentido, realização e relacionamentos positivos, e ela sugeriu ainda que é possível determinar o que torna uma pessoa feliz. No entanto, é importante lembrar que todos esses fatores são formados por fatores subjetivos que variam de pessoa para pessoa (Camalionte \& Boccalandro, 2017).

É importante descrever a relação de bem-estar presente em um relacionamento amoroso, seja ele conjugal ou informal, por meio de relacionamentos de longo prazo e de apoio para aumentar a autoestima pessoal e a intimidade, o casamento é entendido como um elemento diretamente relacionado à promoção da felicidade, e que faz parte da biblioteca de relacionamento interpessoal e é considerado muito importante para a construção da felicidade, envolve fatores importantes no nível humano, pois os relacionamentos de longo prazo tornam o sistema de casamento um refúgio e um mecanismo para apoiar situações estressantes (Campos, 2017).

Nessa perspectiva, o presente estudo teve como principal objetivo compreender a construção da personalidade na perspectiva da Psicologia Positiva e de que maneira os atributos de personalidade são favoráveis para a construção de bemestar e satisfação nos relacionamentos amorosos. Tem-se como pergunta norteadora "Quais características de personalidade são potencializadoras para construção de relacionamentos felizes?" A hipótese levantada para a realização da pesquisa sugeriu que características de personalidade, como os fenômenos do otimismo, espiritualidade, imagem corporal e criatividade estão associados à construção de relacionamentos felizes.

Este estudo justifica-se pela sua relevância social, considerando que se trata de uma temática presente no cotidiano dos relacionamentos amorosos e possui uma intrínseca relação com o bem estar subjetivo das pessoas que compõem essa relação. Ademais, os resultados dessa pesquisa poderão contribuir diretamente para a comunidade científica, tendo em vista que é uma temática pouco abordada na literatura e ainda será de extrema importância, como informação e orientação à comunidade leitora acerca do caminho a ser trilhado na busca da felicidade e do bem-estar dentro de um relacionamento amoroso.

\section{Metodologia}

O presente estudo trata-se de uma pesquisa bibliográfica do tipo revisão narrativa, descritiva e exploratória, com abordagem qualitativa. Este método foi escolhido por possibilitar a síntese, análise e levantamento do conhecimento científico já produzido sobre o tema "Relacionamentos amorosos e seus constituintes para a felicidade".

Acerca da pesquisa bibliográfica, Lakatos (2018) descreve como um levantamento de todas as referências publicadas, incluindo livros, revistas, publicações individuais e coletivas, que permite aos pesquisadores acesso direto a todo o conteúdo concluído acerca do tópico. Também pode ser considerada a primeira etapa de toda pesquisa científica. Enquanto que revisão narrativa são uma ampla gama de publicações adequadas para descrever e discutir desenvolvimentos ou "estado da arte", em um determinado tópico de uma perspectiva teórica ou contextual. Incluem, principalmente, a análise de documentos publicados 
em livros, revistas impressas e / ou eletrônicas e trazem a interpretação dos autores e análises críticas pessoais (Rother, 2007).

O objetivo principal da pesquisa descritiva é descrever as características de uma determinada população ou fenômeno. São inúmeros os estudos que podem ser classificados como descritivos, e uma de suas funções mais importantes é o manuseio de procedimentos de coleta de dados padronizada (Menezes, Duarte, Carvalho, \& Souza, 2019). A pesquisa exploratória conforme Gil (2008) é desenvolver, esclarecer e modificar conceitos e ideias para levantar questões mais complexas, hipóteses precisas para pesquisas futuras. Geralmente envolvem pesquisas bibliográficas e de literatura, entrevistas não padronizadas e estudos de caso. Procedimentos de amostragem e técnicas quantitativas de coleta de dados geralmente não se aplicam a esse método.

A pesquisa qualitativa envolve principalmente a pesquisa e a análise do mundo empírico em seu modo natural. Neste método, aprecia-se o contato direto e de longo prazo do pesquisador com a situação da pesquisa e as circunstâncias que está sendo estudada (Zanella, 2013).

Para a realização desta pesquisa foi feito um levantamento bibliográfico durante o período de 2020, para seleção dos estudos nas bases de dados on-line: Scientific Electronic Library Online (SciELO); Periódicos Eletrônicos de Psicologia (PePSIC) e Portal Regional BVS. Em decorrência da escassez de referências sobre o tema proposto, não foi utilizada uma delimitação temporal. Foram utilizados os seguintes descritores: Psicologia Positiva; Personalidade e Relacionamento conjugal, com a forma booleana "AND”. Os critérios de inclusão para a seleção dos estudos foram: artigos completos referentes à temática pesquisada, em Língua Portuguesa, indexados nas bases escolhidas. Os critérios para exclusão dos estudos foram: resumos, congressos e textos incompletos, digressão da temática e duplicação de artigos.

Após a coleta de dados, foi realizada uma análise dos objetivos e resultados de todos os estudos a fim de obter os pontos de partida e desfechos que mais fundamentavam o tema em questão. Em seguida foi realizada a leitura de todo material escolhido e reunidas as principais informações que se adequavam ao tema proposto buscando estabelecer uma compreensão e discussão sobre o assunto em questão.

\section{Resultados e Discussão}

Foi realizada uma busca livre na literatura cientifica sobre os artigos que mais se adequavam ao tema proposto. Os constructos predominantemente investigados nas pesquisas cientificas da Psicologia Positiva são a felicidade, bem-estar, otimismo, resiliência, cognitivos motivacionais e os traços de personalidade. A investigação nesse último domínio, que é objeto de estudo desta pesquisa, tem sido potencializada para a promoção da saúde e bem-estar do sujeito em sua constituição intraindividual e social. A Psicologia Positiva apresenta a personalidade por meios das forças de caráter e virtude. Martin Seligman, o principal teórico da Psicologia Positiva, refere-se aos traços de personalidade como características negativas ou positivas que aparecem repetidamente em diferentes situações, enquanto forças e virtudes são características positivas que levam a bons sentimentos e satisfação (Seligman, 2004).

É importante expressar que, na perspectiva da Psicologia Positiva, os atributos conceituais da personalidade apresentam as características do sujeito como recursos pessoais, denominados virtudes e forças de caráter. Frente a essa compreensão, as virtudes e forças de caráter podem ser caracterizadas como uma área de investigação da Psicologia Positiva que se concentra nas qualidades positivas do ser humano, as principais referências da Psicologia Positiva propõem 24 forças agrupadas em seis virtudes amplas (Dametto \& Noronha, 2019).

Nesse sentido, as virtudes e forças de caráter são colocadas, de acordo com a Psicologia Positiva, como constructos da personalidade. É importante compreender que ser uma pessoa virtuosa é demonstrar por vontade própria todas ou pelo menos a maior parte das seis virtudes, sendo que são vários os caminhos para alcançá-las. Enquanto que esses caminhos de força, ao contrário da virtude, cada força é mensurável e pode ser adquirida, pois, a força é um traço, uma característica psicológica, 
possível de ser observada em várias situações e ocasiões e, de modo geral, geram boas consequências (Seligman, 2004).

As virtudes são definidas como as características básicas do ser humano, divididas em seis categorias: sabedoria, conhecimento, coragem, humanidade, justiça, temperança e transcendência. Essas características estão baseadas no processo evolutivo e são um meio para resolver tarefas importantes e necessárias à sobrevivência humana, portanto, essas virtudes devem estar refletidas nos valores pessoais, ou seja, nos conceitos morais (Alves \& Ambiel, 2018). Frente a isso, percebe-se a importância da busca pelo fortalecimento dessas virtudes com o objetivo de uma vida mais qualificada e satisfatória.

Segundo Peterson e Seligman (2004) as virtudes são classificadas em seis categorias e 24 forças de caráter, quais sejam: Sabedoria e Conhecimento (criatividade, curiosidade, pensamento crítico, amor ao aprendizado e sensatez); Coragem (bravura, perseverança, autenticidade e vitalidade); Humanidade (amor, bondade e inteligência social); Justiça (imparcialidade e liderança); Temperança (perdão, modéstia, prudência e autorregulação); Transcendência (apreciação o belo, gratidão, esperança, humor e espiritualidade). São várias as características que podem descrever as virtudes e forças de caráter, como pode ser observado no Quadro 1 a seguir.

Quadro 1. Classificação das virtudes e forças de caráter.

\begin{tabular}{|c|c|}
\hline VIRTUDES & FORÇAS DE CARÁTER \\
\hline $\begin{array}{c}\text { Sabedoria e Conhecimento } \\
\text { (Forças cognitivas que implicam a aquisição e utilização do } \\
\text { conhecimento) }\end{array}$ & $\begin{array}{l}\text { Criatividade (originalidade, ingenuidade) } \\
\text { Curiosidade (interesse, busca da novidade, abertura a experiência) } \\
\text { Julgamento (pensamento crítico, "mente aberta") } \\
\text { Gosto pela aprendizagem } \\
\text { Perspectiva (sabedoria) }\end{array}$ \\
\hline $\begin{array}{l}\text { Coragem } \\
\text { (Forças emocionais que envolvem o exercício de querer } \\
\text { atingir objetivos perante a obstáculos externos ou internos) }\end{array}$ & $\begin{array}{l}\text { Bravura (valor) } \\
\text { Perseverança (persistência) } \\
\text { Honestidade (autenticidade, integridade) } \\
\text { Entusiasmo (vitalidade, vigor e energia) }\end{array}$ \\
\hline $\begin{array}{c}\text { Humanidade } \\
\text { (Forças interpessoais que envolvem o "cuidar e ajudar" os } \\
\text { outros) }\end{array}$ & $\begin{array}{l}\text { Amor (capacidade de amar e ser amado) } \\
\text { Bondade (generosidade, cuidado, compaixão, altruísmo, gentileza) } \\
\text { Inteligência Social (inteligência emocional, inteligência pessoal) }\end{array}$ \\
\hline $\begin{array}{c}\text { Justiça } \\
\text { (Forças cívicas que subjazem uma vida em comunidade } \\
\text { saudável) }\end{array}$ & $\begin{array}{l}\text { Trabalho em equipe (cidadania, responsabilidade social, lealdade) } \\
\text { Equidade } \\
\text { Liderança }\end{array}$ \\
\hline $\begin{array}{c}\text { Temperança } \\
\text { (Forças que protegem contra o excesso) }\end{array}$ & $\begin{array}{l}\text { Perdão } \\
\text { Humildade (modéstia) } \\
\text { Prudência } \\
\text { Autorregulação (autocontrole) }\end{array}$ \\
\hline $\begin{array}{c}\text { Transcendência } \\
\text { (Forças que promovem significado a vida) }\end{array}$ & $\begin{array}{l}\text { Valorização da beleza e Excelência (admiração, maravilha, elevação) } \\
\text { Gratidão } \\
\text { Esperança (otimismo, mentalidade orientada para o futuro) } \\
\text { Humor (brincadeira) } \\
\text { Espiritualidade (religiosidade, fé, propósito) }\end{array}$ \\
\hline
\end{tabular}


A Psicologia Positiva, por meio das virtudes e forças de caráter apresentadas no quadro 1, mostra que a personalidade é uma construção plural, porém sempre atribuindo dimensões de responsabilidades frente ao florescer dos atributos que se somam à constituição do ser e as forças de caráter necessárias para a atribuição de cada uma dessas virtudes.

Todo ser humano presenta em sua personalidade forças de caráter e virtudes que são vieses para o desenvolvimento do ser e seu florescimento, na perspectiva de prosperar para uma vida de bem-estar psicológico. Assim, é essencial destacar que as virtudes não andam sozinhas, para que a pessoa consiga trabalhar essas virtudes, precisaria ter conhecimento das forças de caráter que possui, pois as forças de caráter são qualidades pessoais, positivas e mensuráveis da função mental de uma pessoa e são entendidas como elementos da virtude, por exemplo, para que um indivíduo tenha as virtudes da sabedoria e do conhecimento, ele precisa mostrar algumas vantagens, como criatividade, pensamento crítico, amor pelo aprendizado e sabedoria (Alves \& Ambiel, 2018).

Diante das informações descritas acerca das virtudes, forças de caráter e seus significados, considera-se indispensável identificar e descrever as formas pelas quais as virtudes podem contribuir e/ou auxiliar no funcionamento ótimo da vida a dois. Considerando que cada força de caráter é elemento de uma virtude, serão apresentadas as forças que, consequentemente, representarão uma virtude na categorização a seguir.

Os fenômenos do otimismo, espiritualidade e criatividade, contribuem para uma compreensão dinâmica da personalidade e articulam-se como fatores de proteção e promoção para um relacionamento mais equilibrado e satisfatório, ou seja, são atributos fundamentais para gerar relacionamentos sociais saudáveis. Esses três elementos são fundamentais para a satisfação dos relacionamentos amorosos e conjugais, já que um pensamento otimista, a vivência, a recriação e o cuidado dentro de uma relação amorosa, são estratégias essenciais para uma vida a dois com qualidade (Schlosser, 2014).

Estima-se que, além de apresentar comportamentos mais saudáveis relacionados à melhoria da qualidade de vida, os otimistas também têm maior probabilidade de se adaptar às adversidades e menor chance de desenvolverem transtornos mentais. Tendo uma atitude otimista em relação à vida, vivenciando e remodelando relacionamentos ao longo do tempo, cuidar de si e do seu parceiro também é uma estratégia importante para satisfazer sua experiência amorosa e matrimonial (Santos \& Faro, 2020).

De acordo com Hoffmann e Costa (2019) acerca da espiritualidade, força capaz de ampliar a virtude transcendência, e sua contribuição para um relacionamento, destaca-se que é um elemento que torna o casal mais próximo, gera gentileza e estimula sentimentos positivos, manter a intimidade espiritual em conversas sobre temas espirituais e frequentar programas religiosos são aspectos que ajudam a aumentar o compromisso e a satisfação com o casamento, e encorajam os parceiros a permanecerem amigos e evitar emoções negativas.

A criatividade, como componente da virtude "Sabedoria e Conhecimento", pode contribuir na vida do casal a partir da premissa de que um casal criativo suporta o tempo, o tédio, a rotina e encontra diversas maneiras de viver, de amar e de se relacionar, e nesse contexto a criatividade pode ser utilizada como um fator de saúde e mudança, pode penetrar na vida conjugal e nas relações interpessoais saudáveis e favorecer o melhor uso do potencial criativo de cada uma das partes envolvidas, com isso a vida de um casal criativo é profunda e verdadeira e gera sensibilidade da parte dos cônjuges (Silveira, 2007).

O perdão, força elemento da virtude "temperança", constitui-se como um importante componente na busca de um relacionamento amoroso saudável, essencial para os momentos de reconciliação e sobreposição dos sentimentos negativos que podem surgir a partir dos atritos dentro da relação. Perdoar é libertar-se de mágoas desnecessárias, que trazem desconforto ou até mesmo um adoecimento emocional. Perdoar é ser capaz de dar à relação uma segunda chance, livre de cargas do passado.

Nessa perspectiva, Limeira e Feres-Carneiro (2019) acrescentam que o perdão é essencial para a reconciliação e o estabelecimento de um relacionamento conjugal saudável, é importante focar no perdão, para que o processo de reconciliação 
seja benéfico para todos e traga alegria, saúde emocional e motivação para o desenvolvimento pessoal. Para lidar com os conflitos diários dos casais, um estilo de vida de perdão e cuidados emocionais deve ser estabelecido a fim de construir um relacionamento mais resiliente e saudável.

Um dos objetivos da Psicologia Positiva é apresentar os benefícios das virtudes e forças de caráter e como esses atributos podem contribuir para a construção de uma vida proativa em seu desenvolvimento positivo, favorecendo assim os relacionamentos conjugais. Entre os fenômenos indicativos de relacionamentos conjugais saudáveis, a literatura salienta que otimismo, criatividade, espiritualidade e perdão são constructos capazes de favorecer o florescimento do ser intraindividual e conjugal e auxiliar em um modo de vida mais leve e positivo para o indivíduo e suas relações.

\section{Considerações Finais}

A presente pesquisa identificou as características de personalidade abordadas pela Psicologia Positiva e sua relevância para a construção de relacionamentos felizes e satisfatórios. De acordo com os achados da pesquisa, é possível afirmar que os constructos da personalidade apresentados pela Psicologia Positiva e denominados virtudes e forças de caráter são capazes de contribuir de forma considerável para o estabelecimento de bem-estar e qualidade na relação amorosa, desde que sejam trabalhadas e desenvolvidas nessa perspectiva de amplificação intraindividual e conjugal.

O otimismo, a criatividade, a espiritualidade e o perdão representam, nesse caso, os elementos das virtudes capazes de trazer significativas contribuições para uma vida a dois mais produtiva, satisfatória, e propensa a se sustentar diante das adversidades encontradas no dia a dia.

A literatura apresenta que os sujeitos que buscam desenvolver suas habilidades virtuosas e forças de caráter, tendem a amplificar a sua capacidade de resolução de conflitos, aderindo à sua personalidade características potencializadoras do eu e conseguem olhar para as situações conflituosas de uma outra perspectiva para resolver aquele problema sem que gere algum adoecimento, tornando seus relacionamentos mais qualificados, duradouros e prazerosos. Entretanto ainda são necessários mais estudos acerca da temática, pois através de pesquisas e publicações pode-se fomentar uma sustentação teórica rica e abrangente para uma área pouco explorada.

\section{Referências}

Alves, B. P., \& Ambiel, R. A. M. (2018). Escala de forças de caráter: relações com instrumentos de avaliação de afetos e interesses profissionais. Est. Inter. Psicol., 9(2), 04-20.

Camalionte, L. G., \& Boccalandro, M. P. R. (2017). Felicidade e bem-estar na visão da Psicologia Positiva. Bol. Acad. Paul. Psicol., 37(93), $206-227$.

Campos, S. O., Scorsolini-comin, F., \& Santos, M. A. (2017). Transformações da conjugalidade em casamentos de longa duração. Psicol. clin. $29(1)$, 69-89.

Dametto, D. M., \& Noronha, A. P. P. (2019). Construção e validação da escala de forças de caráter para jovens (CSS-Youth). Paidéia, 29(e2930), 01-10.

Figueiras, M. M. (2017). A Relação entre Bem-Estar Psicológico, Autoestima e Felicidade: Diferenças entre alunos do ensino superior privado e alunos do ensino superior público em Portugal (Dissertação de mestrado). Universidade Fernando Pessoa. Porto, Portugal. https://bdigital.ufp.pt/bitstream/10284/6209/1/DM_\%20Marcelo\%20Figueiras.pdf

Gil, A. C. (2008). Métodos e técnicas de pesquisa social. (6a ed.), Atlas.

Hoffmann, E. F., \& Costa, C. B. (2019). Associações entre religiosidade - espiritualidade e as relações conjugais: estudo de revisão sistemática. Contextos Clínic, 12(2), 533-559.

Lakatos, E. M. (2017). Fundamentos de metodologia científica. (8a ed.), Atlas.

Limeira, M. I. C. A., \& Feres-Carneiro, T. (2019). O papel do perdão na reconciliação: uma análise qualitativa das repercussões no recasamento com o excônjuge. Contextos Clínic, 12(3), 822-842.

Menezes, A. H. N., Duarte, F. C., Carvalho, L. O. R., \& Souza, T. E. S. (2019). Metodologia científica: teoria e aplicação na educação a distância. Petrolina: Universidade Federal do Vale do São Francisco.

Nunes, P. (2008). Psicologia Positiva. Psicologia.pt, 01-49. 
Research, Society and Development, v. 10, n. 9, e6810917750, 2021

(CC BY 4.0) | ISSN 2525-3409 | DOI: http://dx.doi.org/10.33448/rsd-v10i9.17750

Peterson, C., \& Seligman, M. E. P. (2004). Character strengths and virtues: A handbook and classification. American Psychological Association. Oxford University Press.

Rother, E. T. (2007). Revisão sistemática X revisão narrativa. Acta Paulista de Enfermagem, 20(2), 5-6.

Santos, L., \& Faro, A. (2020). Otimismo: teoria e aplicabilidade para a psicologia. Rev. Psicol. Saúde, 12(2),123-139.

Schlosser, A. (2014). Interface entre saúde mental e relacionamento amoroso: um olhar a partir da Psicologia Positiva. Pensando fam., 18(2), 17-33.

Seibel, B. L., Sousa, D., \& Koller, S. H. (2015). Adaptação Brasileira e Estrutura Fatorial da Escala, 240 item VIA Inventário de Forças. Psico-USF, 20(3), 371-383.

Seligman, M. E. P. (2012). Florescer, uma nova compreensão sobre a natureza da felicidade e do bem-estar. Objetiva.

Seligman, M. E. P. (2004). Felicidade Autêntica: Usando a nova Psicologia Positiva para a realização permanente. Objetiva.

Silveira, T. M. (2007). O papel da criatividade nas relações conjugais: os limites do" eu" e os limites do" nós". Revista IGT na Rede, 4(7),199-207.

Zanella, L. C. H. (2013). Metodologia de pesquisa. (2a ed.), Departamento de Ciências da Administração/ UFSC. 